\title{
Occurrence of ocular melanoma thirteen years after skin melanoma: two separate primaries or metastatic disease? A case solved with $N R A S$ and $C D K N 2 A$ (INK4A-ARF) mutational analysis
}

\author{
Heidi V. N. Küsters-Vandevelde • Jan E. E. Keunen • \\ Pieter Wesseling • Marian A. J. Verdijk • \\ Marjolijn J. L. Ligtenberg • Willeke A. M. Blokx
}

Received: 28 November 2007 / Accepted: 29 November 2007 / Published online: 17 January 2008

(C) The Author(s) 2007

\begin{abstract}
The differential diagnosis between primary uveal melanoma and cutaneous melanoma metastasis in the eye may be difficult, both clinically and histologically. We report successful application of combined mutational analysis of the $N R A S$ and the $C D K N 2 A$ gene to discriminate between these two entities. The patient had a history of a superficial spreading cutaneous melanoma of the left shoulder. Nine years later, she developed a lymph node metastasis in the left axilla, and 13 years later she presented with an atypical, pigmented tumor in the uvea. Histologically, the origin of the uveal melanoma could not be determined with certainty. We performed molecular analysis on the skin melanoma, the lymph node metastasis and the uveal melanoma. We detected an NRAS codon 61 mutation (c.182A $>\mathrm{G}$, p.Gln61Arg) in all three tumor specimens. This mutation was absent in the normal control tissue of the patient, thereby excluding a germline mutation. To confirm a clonal relationship between the tumors, we
\end{abstract}

H. V. N. Küsters-Vandevelde $(\bowtie) \cdot$ P. Wesseling •

M. A. J. Verdijk • M. J. L. Ligtenberg • W. A. M. Blokx

Department of Pathology C66,

Canisius Wilhelmina Hospital,

P.O. Box 9015, 6500 GS Nijmegen,

The Netherlands

e-mail: H.vandevelde@pathol.umcn.nl

J. E. E. Keunen

Department of Ophthalmology,

Radboud University Nijmegen Medical Centre,

Nijmegen, The Netherlands

\section{J. L. Ligtenberg}

Department of Human Genetics,

Radboud University Nijmegen Medical Centre,

Nijmegen, The Netherlands also performed $C D K N 2 A$ mutational analysis. We detected a $C D K N 2 A$ mutation ((p16) c.238C $>$ T, p.Arg80X, (p14) c. $404 \mathrm{C}>\mathrm{T}$, p.Pro135Leu)) in the tumor samples, but not in the normal control tissue of the patient. We concluded that the uveal melanoma is a metastasis from the cutaneous melanoma removed 13 years before.

Keywords Melanoma $\cdot$ Metastasis $\cdot$ Uveal $\cdot N R A S$. CDKN2A

\section{Introduction}

Especially in case of two cutaneous melanomas or a combination of an ocular and cutaneous melanoma, the clinical and histological differential diagnosis between a second primary melanoma and a melanoma metastasis can be very difficult, as both organs show a relative high frequency of primary melanomas. The commonest primary intraocular malignancy of adults is uveal melanoma [10]. Metastatic melanoma to the eye is rare and generally occurs in patients with widespread metastatic disease [17]. The most common site of metastatic cutaneous melanoma involvement of the eye is the choroid (46\%). Metastatic melanoma to the vitreous and anterior chamber is rare (11$18 \%$ of all intraocular cutaneous melanoma metastases) [6]. Differentiation between a primary uveal melanoma and an intraocular metastasis is of prognostic and clinical importance, as intraocular metastases imply systemic spread and have a poor prognosis with a patient survival rarely exceeding more than 1 year [17].

Mutations in $B R A F$ and less frequently in NRAS are involved in cutaneous melanoma development $[4,11]$. In 
Table 1 Primers used in the analysis of $C D K N 2 A, B R A F, N R A S$ and $H R A S$, and the size of their PCR products

\begin{tabular}{llll}
\hline $\begin{array}{l}\text { Exon } \\
\text { CDKN2A (INK4a-ARF)* }\end{array}$ & Forward & Reverse & Size PCR product (bp) \\
\hline 1B & TCAGGGAAGGCGGGTGCGCG & GCCGCGGGATGTGAACCAC & 244 \\
$1 \mathrm{~B}$ & CGCCGCGAGTGAGGGTTTT & CACCGCGGTTATCTCCTCC & 263 \\
1A & GAGAGGGGGAGAGCAGGCAG & GCACCTCCTCTACCCGACC & 122 \\
$1 \mathrm{~A}$ & GGAGCAGCATGGAGCCTTC & AGTCGCCCGCCATCCC CTG & 177 \\
2 & AGCTTCCTTTCCGTCATGC & GCAGCACCACCAGCGTGTC & 202 \\
2 & AGCCCAACTGCGCCGACCC & CCAGGTCCACGGGCAGACG & 146 \\
2 & TGGACGTGCGCGATGCCTG & GGAAGCTCTCAGGGTACAAATTC & 188 \\
3 & CGGTAGGGACGGCAAGAGAG & GAGGGACCTTCGGTGACTGATG & 162 \\
$B R A F^{*}$ exon 15 & CCTTTACTTACTACACCTCAG & AAAAATAGCCTCAATTCTTAC & 197 \\
$N R A S^{*}$ exon 2 & GGTTTCCAACAGGTTCTTGC & TGGGTAAAGATGATCCGACA & 240 \\
$N R A S^{*}$ exon 3 & GATTCTTACAGAAAACAAGTGG & TAATGCTCCTAGTACCTGTAGAG & 230 \\
$H R A S^{*}$ exon 3 & CTGCAGGATTCCTACCGGA & ACTTGGTGTTGTTGATGGCA & 196 \\
\hline
\end{tabular}

*All forward primers contained the M13 consensus sequence TGT AAA ACG ACG GCC AGT at the 5' end. All reverse primers contained the M13 consensus sequence CAG GAA ACA GCT ATG ACC.

contrast, in primary uveal melanomas, $B R A F$ and $N R A S$ do not appear to be involved in tumorigenesis $[3,8,15]$.

$C D K N 2 A$ mutations have been detected in cutaneous melanoma, both in sporadic melanoma and especially in familial melanoma, with frequencies ranging up to $30 \%$ for the latter [16]. Both in primary sporadic ocular melanoma and in familial cases of primary uveal melanoma, $C D K N 2 \mathrm{~A}$ mutations are very rarely detected $(5 \%)[9,14]$.

We present a case of a rare and relatively late occurrence of an intraocular melanoma metastasis 13 years after development of a primary cutaneous melanoma, and illustrate the diagnostic use of $R A S$ and $C D K N 2 A$ mutational analysis.

\section{Clinical history}

In November 2005, a 47-year-old caucasian female presented to her local ophthalmologist because of mouches volantes in her left eye. She had a history of a superficial spreading cutaneous melanoma of the left shoulder (Clark level IV) removed in 1994, and a lymph node metastasis in the left axilla excised in 2003. Since then no systemic metastasis was detected at annual control. Visual acuity was $20 / 20$ in both eyes and the vitreous of the left eye showed some opacities. These opacities increased gradually over a 2-month period, but resolved spontaneously thereafter. In July 2006, she again developed complaints of recurrent mouches volantes. The diagnosis revealed uveitis, and the patient was treated with local steroids. However, local treatment was not effective and the patient was referred to our center. Examination of the right eye revealed no abnormalities. The visual acuity in the left eye was 20/40, and non-pigmented cells were noticed in the anterior chamber, along with a vitreous haze. As an extensive uveitis screening revealed no abnormalities, intraocular metastasis of the cutaneous melanoma removed 13 years earlier was suspected. A focal, white, and preretinal condensation of vitreous cells developed within a few weeks and some pigmented cells became visible in the vitreous. Subsequently, the intraocular pressure increased to $25 \mathrm{mmHg}$ due to non-pigmented cells in the anterior chamber. A diagnostic vitrectomy was performed. Vitreous samples were positive for malignant melanoma cells. A total body examination excluded systemic metastasis outside the eye. Progressive worsening of the condition of the left eye turned into a visual acuity of $20 / 80$ and a secondary glaucoma up to $50 \mathrm{mmHg}$ occurred, refractive to topical medication. On patient's request, no eye salvaging treatment such as external beam radiation was performed, and the affected eye was enucleated. Histological examination of the eye ball revealed an atypical melanocytic lesion in the iris and ciliary body, with extension under the retina and invasion of the sclera. Although histologically the growth pattern was consistent with primary uveal melanoma, an intraocular metastasis could not be excluded with certainty. As discrimination between second primary and metastatic melanoma was of prognostic importance, we decided to perform mutational analysis for the $B R A F, N R A S$, $H R A S$, and $C D K N 2 A$ genes on all tumor samples, as well as on the normal control skin of this patient.

\section{Materials and methods}

$B R A F, N R A S$, and HRAS mutational analysis

DNA isolation was performed as previously described [1, 2]. Briefly, of all tissue samples about three $10-\mu \mathrm{m}$ unstained sections of formalin-fixed paraffin-embedded 


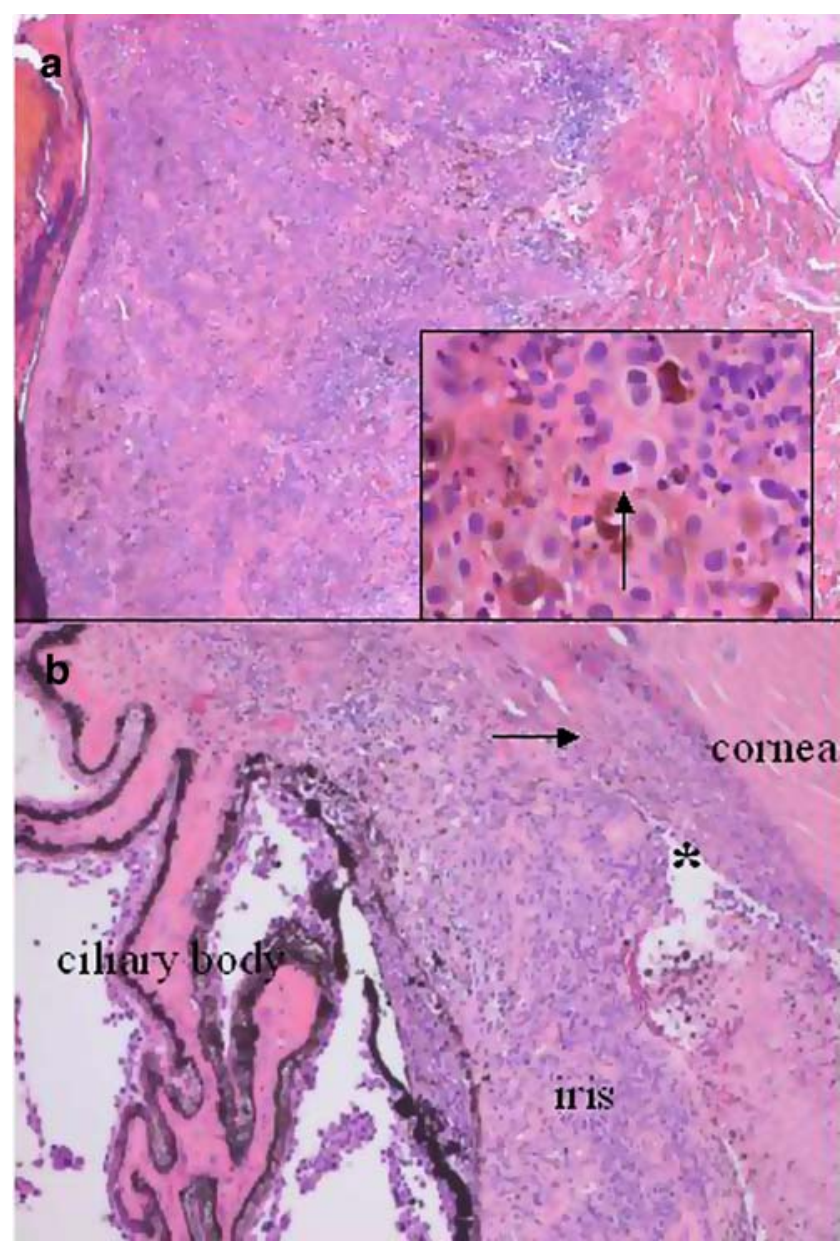

Fig. 1 a. The cutaneous melanoma in 1994 was a superficial spreading melanoma, Breslow thickness $1.5 \mathrm{~mm}$, growing into the reticular dermis (hematoxylin and eosin staining, original magnification $\times 50$ ). The inset shows a detail of the deep portion of the tumor with epithelioid melanocytes, melanin pigment, and mitotic figure (arrow, original magnification $\times 400$ ). b. The intraocular melanoma, located in the ciliary body and iris with invasion of the sclera, anterior angle (asterisk) and canal of Schlemm (arrow) (hematoxylin and eosin staining, original magnification $\times 50$ )

tissue were manually microdissected, using H\&E stained sections as a reference. The tumor cell percentage in the microdissected specimens was estimated as $70 \%$ for the cutaneous and uveal melanoma and as $90 \%$ for the lymph node metastasis. DNA was purified using the DNeasy Blood and Tissue kit (Qiagen). Primer sequences are shown in Table 1. Polymerase chain reaction (PCR) amplification of $B R A F$ exon 15, NRAS exons 2 and 3, and $H R A S$ exon 3 was performed in a total volume of $50-\mu 1$ PCR mix containing $50 \mathrm{ng}$ template DNA, $5 \mu \mathrm{l}$ PCR buffer IV (Integro), $10 \mu \mathrm{dNTP} 0.2 \mathrm{mM}, 6.0 \mu \mathrm{MgCl}_{2} 3.0 \mathrm{mM}$, $0.125 \mu \mathrm{l}$ Taq DNA polymerase (Integro, $5 \mathrm{U} / \mu \mathrm{l}$ ), and $22.9 \mu \mathrm{lmQ}$. An initial denaturation at $92^{\circ} \mathrm{C}$ for $5 \mathrm{~min}$ was followed by 35 cycles of denaturation at $94^{\circ} \mathrm{C}$ for $45 \mathrm{~s}$, annealing at $60^{\circ} \mathrm{C}$ for $45 \mathrm{~s}$, and extension at $72^{\circ} \mathrm{C}$ for $45 \mathrm{~s}$ with a final extension of $5 \mathrm{~min}$ at $72^{\circ} \mathrm{C}$. DNA amplification was performed in a MJ PTC 200 PCR cycler (Biozym). After purification of the PCR products using a Qiaquick PCR purification kit (Qiagen), sequence analysis was performed using BigDye terminator version 2 of Applied Biosystems on an ABI 3730 automatic sequencer (Applied Biosystems).

\section{$C D K N 2 A$ mutational analysis}

The $C D K N 2 A$ mutation analysis was performed as previously described [1, 2]. Briefly, PCR was performed with AmpliTaq Gold DNA Polymerase (Applied Biosystems) using an optimized $\mathrm{MgCl}_{2}$ concentration (varying from 1 to $2 \mathrm{mM}$ ) in a MJ PTC 200 PCR cycler (Biozym). All primers used in the analysis contained either an M13 forward or M13 reverse consensus sequence. All primers and sizes of the PCR products are listed in Table 1. The entire open reading frames of $C D K N 2 A$ were analyzed in DNA isolated from the tumor samples and normal skin.

\section{Results}

Revision of the primary cutaneous melanoma removed in 1994 revealed an asymmetric compound melanocytic lesion with focal ulceration and lack of maturation toward the base of the lesion. The melanocytes showed an epithelioid phenotype with anisokaryosis and prominent nucleoli. Deep mitotic figures were present (Fig. 1). A satellite lesion was present at the junction of the dermis toward the subcutis. Tumor thickness measured $1.5 \mathrm{~mm}$, not including the satellite. Focally, a preexistent nevus was present. Resection margins were at least $2 \mathrm{~mm}$ free of tumor. Revision of the lymph node metastasis in the left axilla that was excised in 2003 revealed a large tumor surrounded by limited preexistent lymphoid tissue. The tumor cells showed morphological resemblance to the cutaneous melanoma cells with an epithelioid phenotype and frequent mitotic figures. In addition, in the lymph node metastasis large areas of necrosis were present.

Histological examination of the eye ball revealed an atypical melanocytic lesion in the iris and ciliary body, with extension under the retina and invasion of the sclera. Tumor cells were also present at the surface of the ciliary body in the anterior angle, and showed invasion in the area containing the canal of Schlemm (Fig. 1). The melanocytic cells showed an epithelioid morphology, with focally spindle cell pattern, marked pleiomorphism of tumor cell nuclei, and occasional mitotic figures. Tumor thickness measured $3 \mathrm{~mm}$.

Molecular analysis of the NRAS gene revealed a codon 61 mutation (c.182A>G, p.Gln61Arg) in all three tumor 
Fig. $2 N R A S$ exon 3 mutation, c. $182 \mathrm{~A}>\mathrm{G}$, p.Gln61 Arg, detected in the primary cutaneous melanoma, the lymph node metastasis and the uveal melanoma. Reverse sequence
Fig. $3 C D K N 2 A$ exon 2 mutation, (p16) c. $238 \mathrm{C}>\mathrm{T}, \mathrm{p}$. Arg80X; (p14) c.404C $>$ T, p.Pro135Leu, detected in the primary cutaneous melanoma, the lymph node metastasis and the uveal melanoma. Forward sequence

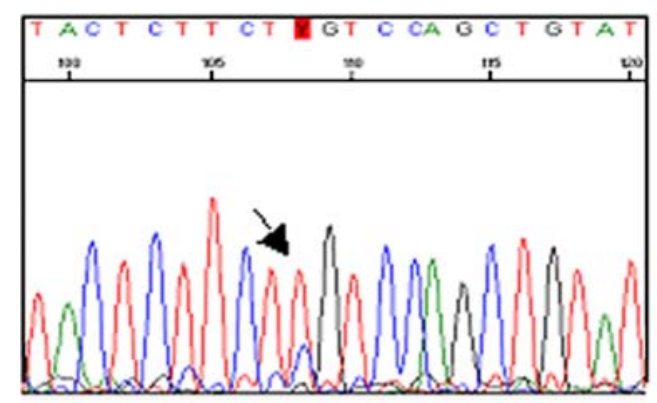

Cutaneous melanoma

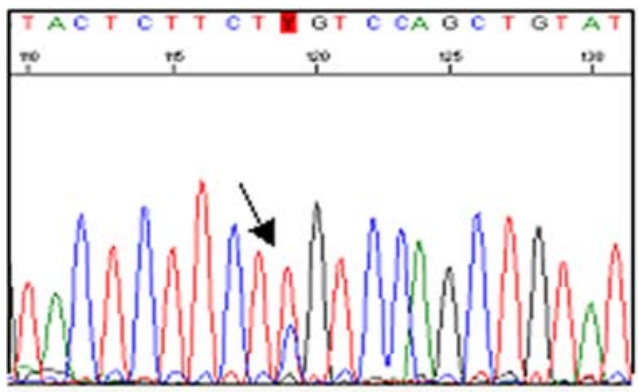

Uveal melanoma

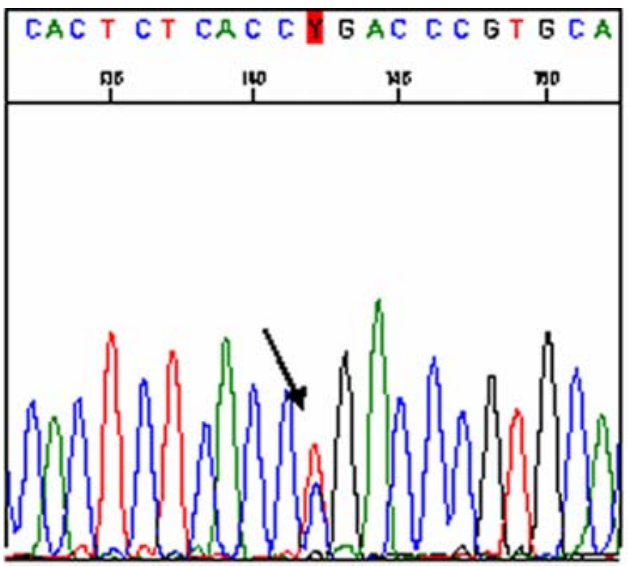

Skin mel anoma

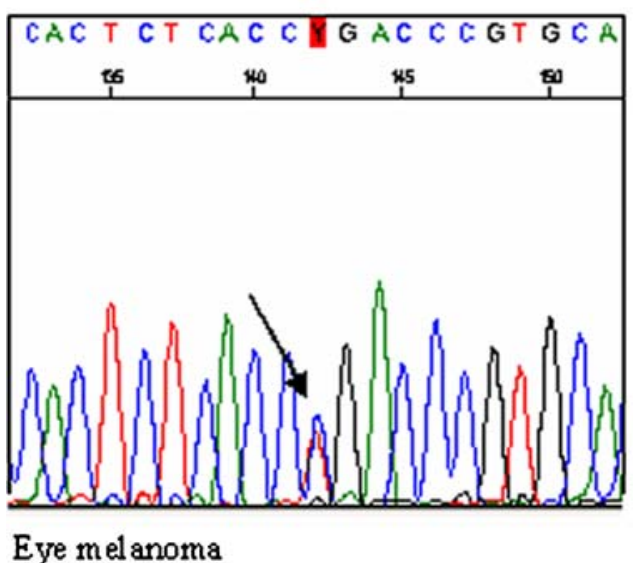

Eye melanoma

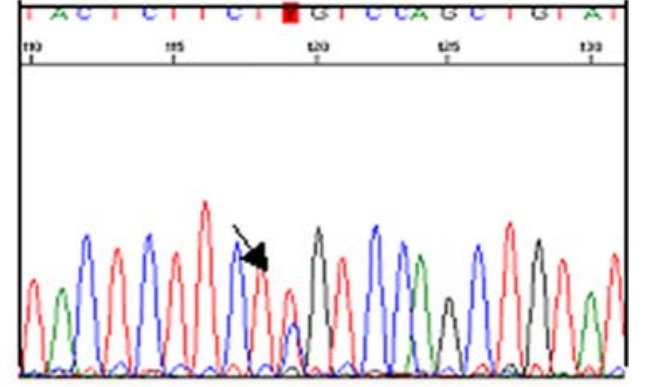

Lymph node metastasis

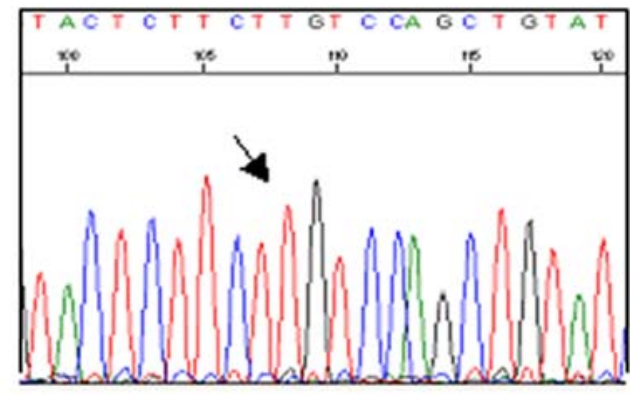

Normal skin

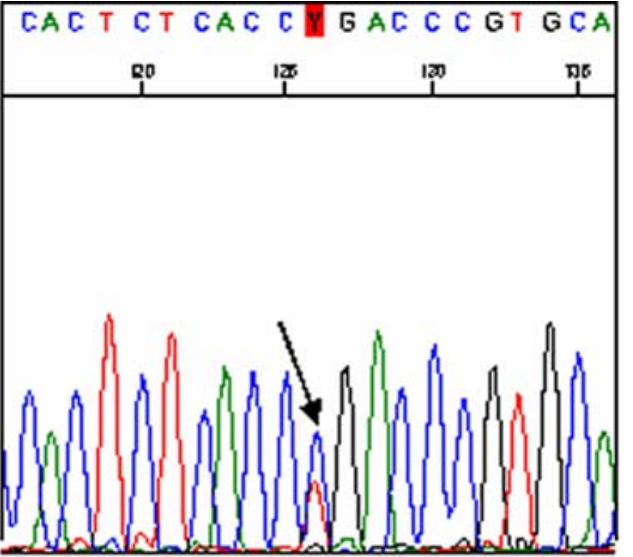

Lymph node metastasis

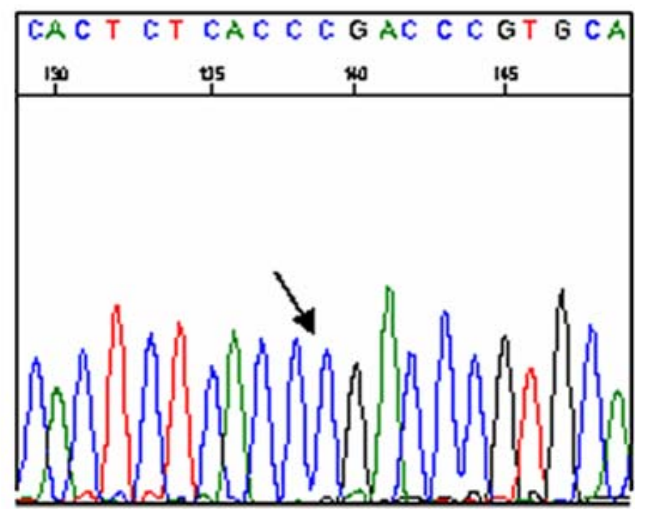

Normal skin 
samples. This mutation was not present in the normal control skin of the patient (Fig. 2). No mutations were detected in $N R A S$ exon 2, BRAF exon 15, and $H R A S$ exon 3. We also performed $C D K N 2 A$ mutational analysis on all samples. A $C D K N 2 A$ mutation ((p16)c.238C $>$ T, p.Arg80X, (p14)c.404C $>$ T, p.Pro135Leu)) was present in all three tumor samples, but absent in the normal control tissue of the patient, indicating that this is a somatic mutation (Fig. 3). It was concluded that the ocular melanoma should be considered as a metastasis of the cutaneous melanoma diagnosed 13 years before.

\section{Discussion}

This case report illustrates that molecular analysis can be very helpful in the differential diagnosis between a second primary melanoma and a melanoma metastasis. We chose for mutational analysis of the $B R A F$ gene and the $R A S$ protooncogenes NRAS and HRAS, because these genes are frequently involved in cutaneous melanocytic lesions, with a reported total mutation frequency for $N R A S$ or $B R A F$ of $82 \%$ in cutaneous melanoma [4]. Furthermore, these mutations are described to occur early in tumorigenesis, before the development of metastasis [4, 11]. We detected in all three tumor samples an NRAS codon 61 mutation (c.182A $>$ G, p.Gln61Arg). NRAS mutations are known to occur in up to $30 \%$ of all cutaneous melanoma cases, the most common being mutations in NRAS codon $61[4,7$, 11]. In contrast, NRAS mutations have been reported to be absent in primary uveal melanoma [3, 15]. The NRAS mutation thus strongly supports the metastatic nature of the intraocular melanoma in our patient. The finding of the same somatic mutation in $C D K N 2 A$ in both cutaneous and intraocular melanoma confirms the metastatic nature of the disease in this patient. In contrast to $N R A S$ mutations that are limited to codon 61 and codon 12 in cutaneous melanoma, somatic CDKN2A mutations are much more tumor specific and thus are not expected to be identical in two different primary melanomas [7]. Furthermore, activating $C D K N 2 A$ mutations are described to be very rare in primary ocular melanomas $[9,13]$.

Eskandarpour et al. reported specific activating mutations in NRAS codon 61 in $95 \%$ of primary hereditary cutaneous melanomas $(20 / 21)$, but in only $10 \%$ of sporadic cutaneous melanomas (1/10) [5]. Therefore, the presence of a somatic $N R A S$ codon 61 mutation might indicate a familial predisposition to melanoma instead of a clonal relationship.

Discriminating between a second primary intraocular melanoma and metastatic disease is of prognostic importance. Intraocular metastases of melanoma are associated with a poor prognosis with a mean interval between diagnosis of the intraocular metastasis and death of
8.8 months [17]. In contrast, survival in patients diagnosed with a primary intraocular melanoma is more variable, with a reported 5-year cumulative survival rate of up to $60 \%$ [12].

In conclusion, this case report illustrates that mutational analysis for the $R A S$ proto-oncogenes and $C D K N 2 A$ can be useful in the clinically relevant, but sometimes difficult, differential diagnosis between a second primary intraocular melanoma and an intraocular metastasis of a cutaneous melanoma.

Conflict of interest statement We declare that we have no conflict of interest.

We declare that the molecular analyses comply with the current laws in The Netherlands.

Open Access This article is distributed under the terms of the Creative Commons Attribution Noncommercial License which permits any noncommercial use, distribution, and reproduction in any medium, provided the original author(s) and source are credited.

\section{References}

1. Blokx WA, Lesterhuis JJ, Andriessen MP, Verdijk MA, Punt KJ, Ligtenberg MJ (2007) CDKN2A (INK4A-ARF) mutation analysis to distinguish cutaneous melanoma metastasis from a second primary melanoma. Am J Surg Pathol 31:637-641

2. Blokx WA, Ruiter DJ, Verdijk MA, de Wilde PC, Willems RW, de Jong EM, Ligtenberg MJ (2005) INK4-ARF and p53 mutations in metastatic cutaneous squamous cell carcinoma: case report and archival study on the use of Ink4a-ARF and p53 mutation analysis in identification of the corresponding primary tumor. Am J Surg Pathol 29:125-130

3. Cruz F III, Rubin BP, Wilson D, Town A, Schroeder A, Haley A, Bainbridge T, Heinrich MC, Corless CL (2003) Absence of BRAF and NRAS mutations in uveal melanoma. Cancer Res 63:57615766

4. Edlundh-Rose E, Egyhazi S, Omholt K, Mansson-Brahme E, Platz A, Hansson J, Lundeberg J (2006) NRAS and BRAF mutations in melanoma tumours in relation to clinical characteristics: a study based on mutation screening by pyrosequencing. Melanoma Res 16:471-478

5. Eskandarpour M, Hashemi J, Kanter L, Ringborg U, Platz A, Hansson J (2003) Frequency of UV-inducible NRAS mutations in melanomas of patients with germline CDKN2A mutations. J Natl Cancer Inst 95:790-798

6. Gunduz K, Shields JA, Shields CL, Eagle RC Jr (1998) Cutaneous melanoma metastatic to the vitreous cavity. Ophthalmology 105:600-605

7. Hocker T, Tsao H (2007) Ultraviolet radiation and melanoma: a systematic review and analysis of reported sequence variants. Hum Mutat 28:578-588

8. Kilic E, Bruggenwirth HT, Verbiest MM, Zwarthoff EC, Mooy NM, Luyten GP, de KA (2004) The RAS-BRAF kinase pathway is not involved in uveal melanoma. Melanoma Res 14:203-205

9. Lamperska K, Mackiewicz K, Kaczmarek A, Kwiatkowska E, Starzycka M, Romanowska B, Heizman J, Stachura J, Mackiewicz A (2002) Expression of p16 in sporadic primary uveal melanoma. Acta Biochim Pol 49:377-385 
10. Lyons CJ, Hungerford J (1990) Detection of ocular malignancies. J R Soc Med 83:165-167

11. Omholt K, Karsberg S, Platz A, Kanter L, Ringborg U, Hansson J (2002) Screening of N-ras codon 61 mutations in paired primary and metastatic cutaneous melanomas: mutations occur early and persist throughout tumor progression. Clin Cancer Res 8:3468-3474

12. Park WL, Jenison-Williams T, Pasqua-Darnell T (2003) Uveal melanoma and poor treatment compliance: an atypical outcome with literature review. Optom Vis Sci 80:344-355

13. Smith JH, Padnick-Silver L, Newlin A, Rhodes K, Rubinstein WS (2007) Genetic study of familial uveal melanoma: association of uveal and cutaneous melanoma with cutaneous and ocular nevi. Ophthalmology 114:774-779
14. Smith JH, Padnick-Silver L, Newlin A, Rhodes K, Rubinstein WS (2007) Genetic study of familial uveal melanoma: association of uveal and cutaneous melanoma with cutaneous and ocular nevi. Ophthalmology 114:774-779

15. Soparker CN, O'Brien JM, Albert DM (1993) Investigation of the role of the ras protooncogene point mutation in human uveal melanomas. Invest Ophthalmol Vis Sci 34:2203-2209

16. Soufir N, Basset-Seguin N (2001) The INK4a-ARF locus: role in the genetic predisposition to familial melanoma and in skin carcinogenesis. Bull Cancer 88:1061-1067

17. Zografos L, Ducrey N, Beati D, Schalenbourg A, Spahn B, Balmer A, Othenin-Girard CB, Chamot L, Egger E (2003) Metastatic melanoma in the eye and orbit. Ophthalmology 110:2245-2256 